\title{
DESAFIOS DIAGNÓSTICOS DE QUADRO MULTISSISTÊMICO DURANTE A PANDEMIA DA COVID-19
}

\author{
DIAGNOSTIC CHALLENGES OF MULTISYSTEMIC CONDITION \\ DURING COVID-19 PANDEMIC
}

\begin{abstract}
Andressa de Deus Mateus', Enéas Van Der Maas Do Bem Filho', Ana Paula Moura de Almeida², Ana Carolina Galvão dos Santos de Araújo1, Ricardo Gomes de Vasconcellos², Daniel Samary Silva Lobato².

${ }^{1}$ Residentes de Dermatologia no Hospital Escola Álvaro Alvim em Campos dos Goytacazes, Rio de Janeiro, Brasil. ${ }^{2}$ Docentes na Faculdade de Medicina de Campos, Campos dos Goytacazes, Rio de Janeiro, Brasil. Instituição no qual o trabalho foi realizado: Hospital Escola Álvaro Alvim. R. Barão da Lagoa Dourada, 409 Centro, Campos dos Goytacazes - RJ, 28035-211.
\end{abstract}

Autor correspondente: Andressa de Deus Mateus. R. Marcílio Dias, número 40, Parque Tamandaré, Campos dos Goytacazes - RJ, 28035-015. Telefone: (27) 99530-1965. E-mail: andmateus@hotmail.com.

\section{RESUMO}

A infecção pelo novo coronavírus (SARS-CoV-2) resultou em uma drástica mudança na saúde mundial. Diversos protocolos hospitalares foram implantados, bem como novas medidas de segurança com afastamento de vários profissionais, dificultando ainda mais os diagnósticos clínicos. O objetivo deste estudo é de apresentar o caso de uma paciente com quadro multissistêmico e diagnóstico histopatológico de parapsoríase em um drástico contexto de saúde pública diretamente afetado pela pandemia da COVID-19.

Palavras-chave: infecções por Coronavírus, diagnóstico, parapsoríase.

\begin{abstract}
Coronavirus (SARS-CoV-2) new infection has resulted in a drastic change in global health. Several hospital protocols were implemented, as well as new safety measures with the removal of several professionals, making clinical diagnoses even more difficult. The objective of this study is to present the case of a patient with a multisystemic condition and histopathological diagnosis of parapsoriasis in a drastic public health context directly affected by the COVID-19 pandemic.

Keywords: Coronavirus infections, diagnosis, parapsoriasis.
\end{abstract}




\section{INTRODUÇÃO}

O primeiro caso de infecção do novo coronavírus 2 da Síndrome Respiratória Aguda Grave (SARS-CoV-2), agente etiológico da doença infecciosa do coronavirus pandêmico, conhecida como COVID19 , foi identificado na cidade de Wuhan na China, em dezembro de 2019. A doença rapidamente se propagou de uma única cidade para o país inteiro e, posteriormente, para outros países, levando a milhares de mortes, sendo prontamente declarada, pela Organização Mundial de Saúde (OMS), emergência na saúde pública de preocupação internacional.1

Frente a esta doença de alta transmissibilidade, o recurso mais prudente foi a recomendação do isolamento social. Nesse contexto, os profissionais médicos precisaram se adequar restringindo o acesso presencial apenas para os pacientes com quadro de maior instabilidade clínica, destacando-se os atendimentos on-line através da telemedicina, modalidade autorizada durante a pandemia. Entretanto, alguns procedimentos, como a realização de exames e cirurgias eletivas, foram adiados.

Já se sabe que a presença de comorbidades está associada a uma pior evolução do quadro da COVID-19, inclusive para óbito e, somado a isso, pacientes portadores de câncer já apresentam maior suscetibilidade a infecções pelo seu estado de imunossupressão causado pela própria malignidade. ${ }^{1}, 2$

A parapsoríase representa um grupo de dermatose incomum, mas não rara, com apresentação clínica variada assemelhando-se muitas vezes à psoríase. A doença engloba duas categorias, parapsoríase em pequenas e em grandes placas, sendo caracterizada por infiltrado mononuclear na derme composto por células T. É muito importante a diferenciação das duas formas pela associação com condições malignas. 4

\section{OBJETIVO}

Relatar um caso que demandou urgência diagnóstica durante a pandemia da COVID-19 e avaliar as dificuldades diagnósticas, bem como o manejo de demandas clínicas neste período.

\section{DESCRIÇÃO DO CASO}

Paciente do sexo feminino, 34 anos, parda, previamente hígida. Encaminhada ao Hospital Escola Álvaro Alvim em Campos dos Goytacazes, Rio de Janeiro para investigação de quadro dermatológico e achado incidental de massa mediastinal.

Na história referia há dois meses surgimento de pápulas e placas hipercrômicas e pruriginosas localizadas em tronco e membros superiores. Procurou atendimento médico em Unidade Básica de Saúde, recebendo tratamento para escabiose. Relata que não houve melhora e que o quadro evoluiu com epigastralgia intensa associada a náuseas, vômitos de conteúdo gástrico, icterícia e perda ponderal, procurando o Pronto Atendimento para nova avaliação médica. Procedeu-se investigação com exames de imagem, mostrando na ultrassonografia de abdome uma vesícula biliar hipodistendida, sem outras alterações e na tomografia (TC) de tórax, abdome e pelve, a formação tecidual com densidade de partes moles e pequenos focos de calcificação de permeio com contornos irregulares, medindo $11,1 \mathrm{x}$ $5,8 \times 3,7 \mathrm{~cm}$ localizada no mediastino anterior, de etiologia a esclarecer. Demais achados dentro da normalidade.

Recebemos a paciente em nosso serviço para internação hospitalar com quadro de icterícia, múltiplas placas e pápulas hipercrômicas de base eritematosa, algumas com crostas, em tronco e membros superiores (Figura 1).

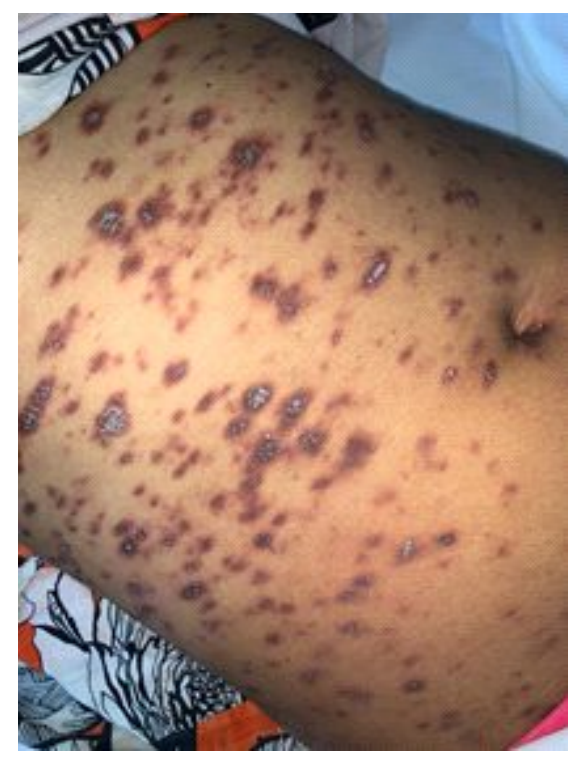

Figura 1. Múltiplas placas hipercrômicas de base eritematosa em tronco, algumas apresentado crostas, medindo entre 2 a $5 \mathrm{~cm}$.

Um dia após a internação foi aventada a possibilidade de COVID-19 por quadro de febre, tosse e dispneia. Realizada TC de tórax que evidenciou infiltrado em vidro fosco envolvendo menos que $25 \%$ do parênquima pulmonar, sendo solicitado isolamento para contato e aerossóis em enfermaria 
destinada para casos suspeitos de COVID-19, onde permaneceu por 7 dias, até resultado de RT-PCR negativo.

Ao retornar para seguimento clínico em enfermaria manteve quadro respiratório, sendo realizada nova TC de tórax que evidenciou infiltrado em vidro fosco acometendo entre $50-70 \%$ do parênquima pulmonar. $A$ paciente foi novamente isolada e iniciado tratamento com Piperacilina/Tazobactam e Dexametasona, permanecendo por 10 dias até posterior sorologia negativa. Foi iniciado uso de Meropenem pela manutenção das queixas respiratórias e neste momento as lesões cutâneas estavam mais extensas, agora acometendo membros inferiores.

Desde a admissão, mostrava em exames laboratoriais: anemia normocítica, leucocitose, hipoalbuminemia, distúrbio da coagulação, elevação de transaminases, bilirrubinas e gama GT, bem como de amilase e lipase. Foram solicitados exames adicionais para investigação de quadro dermatológico: esfregaço de sangue periférico, sem alterações, $H T L V I / I I$, sorologia para hepatite $A, B$ e C, VDRL e anti-HIV todos não reagentes. Apresentou Fator antinuclear 1:160 padrão pontilhado fino. Realizada biópsia cutânea de lesão abdominal, com laudo sugerindo diagnóstico de parapsoríase (Figura $2 \mathrm{~A}$ e $2 \mathrm{~B}$ ), reforçado por imuno-histoquímica. Nova TC

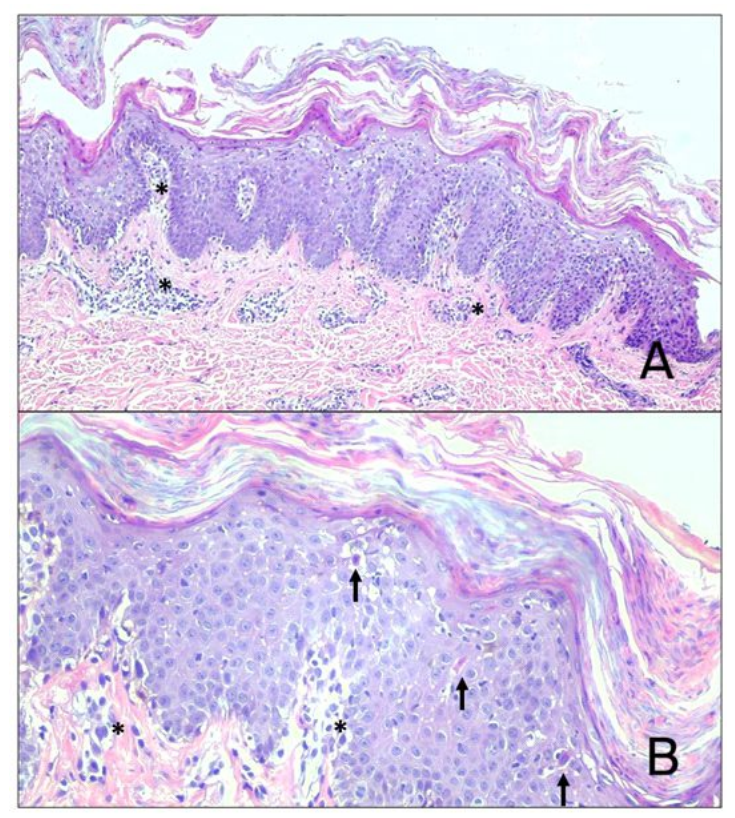

Figura 2. A - Acantose regular, associada a hiperceratose e paraceratose, notando-se áreas de espongiose com exocitose de linfócitos $(*)$, também presentes em derme superficial, predominando ao redor de vasos sanguíneos. (HE 40x). B - Em maior detalhe, áreas de espongiose, ceratinócitos apoptóticos (setas) e linfócitos $(*)$ em exocitose e em derme superficial. (HE 100x). de abdome e pelve, agora contrastada, evidenciou apenas esteatose hepática.

Para investigação de etiologia da massa mediastinal, foram solicitados função tireoideana, beta-HCG e alfafetoproteína, todos sem alterações. Realizada TC de tórax contrastada para melhor definição da massa e posterior abordagem pela cirurgia (Figura 3A e 3B). O exame não mostrou evolução ou crescimento e sugeriu natureza neoplásica.
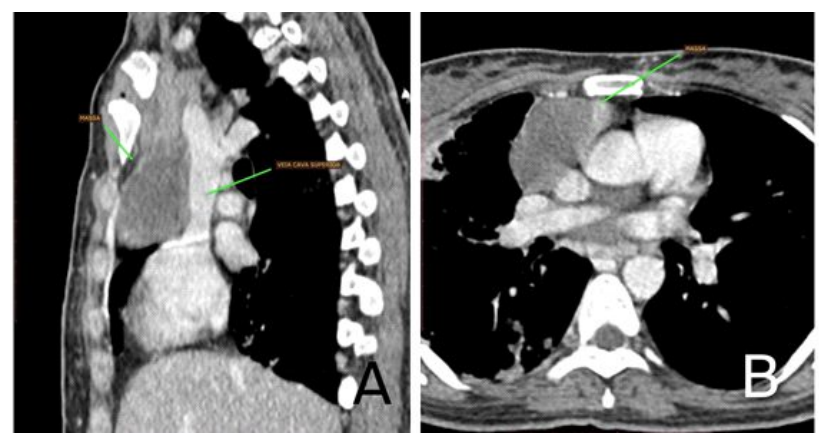

Figura 3. Tomografia contrastada de tórax visualizando massa mediastinal em plano sagital (A) e em plano transversal (B).

Após parecer da cirurgia torácica, foi contraindicada qualquer intervenção da massa mediastinal naquele momento pelos riscos do procedimento frente à debilidade clínica da paciente consequente ao comprometimento respiratório durante seu seguimento. Após 27 dias de internação, a paciente se apresentava assintomática e estável hemodinamicamente, logo, optou-se por conceder alta hospitalar, visto múltiplas intercorrências decorrentes da própria internação.

Cerca de 10 dias após, já em seu domicílio, a paciente evoluiu a óbito, sem conclusão diagnóstica.

\section{DISCUSSÃO}

A parapsoríase foi especificada por Brocq em 1902 para descrever uma manifestação cutânea que clinicamente lembra um quadro de psoríase. O termo engloba um espectro de doenças que varia desde um quadro persistentemente benigno (desordens reativas de células $T$ ) até manifestação de malignidade (linfoma cutâneo de células $T$ ). ${ }^{5}$

A parapsoríase em pequenas placas se apresenta com lesões entre 2 a $5 \mathrm{~cm}$, escamosas crônicas, assintomáticas ou levemente pruriginosas em tronco, acometendo raramente os membros. Essa categoria geralmente representa uma condição benigna. A parapsoríase em grandes placas é uma condição crônica com lesões castanho-amareladas 
maiores do que $5 \mathrm{~cm}$ e apresenta graus variados de atrofia epidérmica, tendo essa condição maior risco de evolução para micose fungóide, um tipo de linfoma cutâneo de células T. ${ }^{5}, 6$

Vale destacar que as dermatoses paraneoplásicas correspondem à segunda síndrome paraneoplásica mais comum, atrás apenas de síndromes endócrinas. Apesar de incomum, há descrição de quadro de parapsoríase em pequenas placas associado a quadro de malignidade interna (câncer pancreatobiliar). ${ }^{7} 0$ padrão clínico das lesões, reforçado pelo diagnóstico histopatológico e imuno-histoquímico nos faz levantar a possibilidade do quadro dermatológico aqui descrito como manifestação paraneoplásica de neoplasia mediastinal. Apesar de não termos a confirmação histopatológica da etiologia da massa torácica, os exames de imagem e o desfecho clínico sugerem fortemente etiologia neoplásica.

Das diversas publicações científicas que reforçam a maior prevalência da COVID-19 em portadores de neoplasias quando comparados à população geral, algumas podem ser destacadas. Yu et al. (2020) referiram uma chance duas vezes maior na suscetibilidade da infecção por pacientes oncológicos e, corroborando com este achado, em revisão, Thuler e Melo (2020) referem que o indivíduo portador de câncer apresenta risco mais elevado para o desenvolvimento da COVID-19 quando comparado à população geral. ${ }^{7,8}$

É irrefutável o impacto da pandemia da COVID-19 neste relato. Além de pesar o risco e o benefício de uma internação hospitalar e, inclusive a possibilidade de infecção pelo SARS-Cov-2 em nosocômio, foi preciso considerar que algumas atividades médicas planejadas foram adiadas, tanto por diretivas nacionais quanto por preocupação dos próprios pacientes.

Diversos serviços criaram estratégias para se adaptar à nova realidade e muitas delas são extremamente benéficas. Destacam-se a telemedicina, a implantação de recursos para avaliação criteriosa das admissões hospitalares e medidas de contenção de transmissão de doenças infecciosas no ambiente intra-hospitalar.

Vivemos um período crítico e, mesmo que lamentavelmente o melhor desfecho não seja sempre obtido, a prática do princípio da não maleficência deve se manter imperioso durante a prática médica. 9

\section{CONCLUSÃO}

A nova e drástica condição da saúde pública gerada pela pandemia da COVID-19 impôs algumas medidas de segurança, como limitações aos profissionais de saúde e diversos protocolos hospitalares. Tudo isso, somado a uma baixa performance status da paciente, não permitiram o acesso à massa mediastinal, impossibilitando a conclusão diagnóstica.

Apesar da paraporíase de grandes placas já ser habitualmente considerada manifestação préneoplásica, em evolução para micose fungóide, a parapsoríase de pequenas placas é designada como doença crônica de evolução benigna, podendo em alguns casos, surgir como manifestação paraneoplásica. A identificação e a intervenção precoce são fundamentais para definição e conduta na parapsoríase, tendo a pandemia da COVID-19 um impacto importante no desfecho clínico do caso relatado.

\section{REFERÊNCIAS}

(1) Wu, Z., \& McGoogan, J. M. (2020). Characteristics of and Important Lessons From the Coronavirus Disease 2019 (COVID-

19) Outbreak in China. JAMA, 323(13), 1239. https://doi.org/10.1001/jama.2020.2648

(2) Al-Quteimat, O. M., \& Amer, A. M. (2020). The Impact of the COVID-19 Pandemic on Cancer Patients. American Journal of Clinical Oncology: Cancer Clinical Trials. Lippincott Williams and Wilkins.

https://doi.org/10.1097/COC.0000000000000712

(3) Lambert, W. C., \& Everett, M. A. (1981). The nosology of parapsoriasis. Journal of the American Academy of Dermatology, 5(4), 373-395. https://doi.org/10.1016/S0190-9622(81)70100-2

(4) Sehgal, V. N., Srivastava, G., \& Aggarwal, A. K. (2007). Parapsoriasis: a complex issue. Skinmed. https://doi.org/10.1111/j.1540-9740.2007.06490.x

(5) Junior, W. B., Chiacchio, N. D., Criado, P. R. Tratado de dermatologia. 3. ed. Rio de Janeiro: Atheneu, 2018.

(6) Philips, C. A., Augustine, P., Kumar, L., Joseph, G., \& Mahadevan, P. (2018). Branch Duct-type Intraductal Papillary Mucinous Neoplasm Presenting as Paraneoplastic Small Plaque Para-psoriasis. Indian Dermatology Online Journal, 9(1), 40-43. https://doi.org/10.4103/idoj.IDOJ_427_16

(7) Yu, J., Ouyang, W., Chua, M. L. K., \& Xie, C. (2020, July 1). SARS-CoV-2 Transmission in Patients with Cancer at a Tertiary Care Hospital in Wuhan, China. JAMA Oncology. American Medical Association. 
https://doi.org/10.1001/jamaoncol.2020.0980

(8) Santos Thuler, L. C., \& De Melo, A. C. (2020). A SARS-CoV-2/COVID-19 em pacientes com câncer. Revista Brasileira de Cancerologia, 66(2). https://doi.org/10.32635/2176-9745.rbc.2020v66n2.970

(9) Mazurik, L., Javidan, A., Higginson, I., Judkins, S., Petrie, D., Graham, C., ... Lang, E. (2020). Early lessons from COVID -19 that may reduce future emergency department crowding. Emergency Medicine Australasia. https://doi.org/10.1111/17426723.13612 